\title{
Beyond the Random-Phase Approximation for the Electron Correlation Energy: The Importance of Single Excitations
}

\author{
Xinguo Ren, Alexandre Tkatchenko, Patrick Rinke, and Matthias Scheffler \\ Fritz-Haber-Institut der Max-Planck-Gesellschaft, Faradayweg 4-6, 14195, Berlin, Germany
}

(Received 21 October 2010; revised manuscript received 21 December 2010; published 12 April 2011)

\begin{abstract}
The random-phase approximation (RPA) for the electron correlation energy, combined with the exactexchange (EX) energy, represents the state-of-the-art exchange-correlation functional within density-functional theory. However, the standard RPA practice-evaluating both the EX and the RPA correlation energies using Kohn-Sham (KS) orbitals from local or semilocal exchange-correlation functionals-leads to a systematic underbinding of molecules and solids. Here we demonstrate that this behavior can be corrected by adding a "single excitation" contribution, so far not included in the standard RPA scheme. A similar improvement can also be achieved by replacing the non-self-consistent EX total energy by the corresponding self-consistent Hartree-Fock total energy, while retaining the RPA correlation energy evaluated using KS orbitals. Both schemes achieve chemical accuracy for a standard benchmark set of noncovalent intermolecular interactions.
\end{abstract}

DOI: 10.1103/PhysRevLett.106.153003

In the quest for finding an "optimal" electronic structure method that combines accuracy and tractability with transferability across different chemical environments and dimensionalities (e.g., molecules, wires or tubes, surfaces, solids), the treatment of exchange and correlation in terms of "exact-exchange plus correlation in the random-phase approximation (EX+cRPA)" [1,2] offers a promising avenue [3-16]. In this approach, part of the exact-exchange energy cancels exactly the spurious self-interaction error present in the Hartree energy. The RPA correlation (cRPA) energy is fully nonlocal, whereby long-range van der Waals (vdW) interactions are included automatically and accurately [17]. Moreover, dynamical electronic screening is taken into account by summing up a sequence of "ring" diagrams to infinite order, making EX+cRPA applicable to small-gap or metallic systems where, for example, Hartree-Fock (HF) plus 2nd-order MøllerPlesset (MP2) perturbation theory [18] breaks down.

The concept of cRPA dates back to the many-body treatment of the uniform electron gas in the 1950s [1,2], and was later formulated [19] within the context of densityfunctional theory (DFT) [20]. Recent years have witnessed a revived interest in EX $+\mathrm{cRPA}$ and its variants in quantum chemistry [3-9], solid state physics [10-12], and surface science [13-15]. Within the framework of Kohn-Sham (KS) DFT, EX+cRPA embodies an orbital-dependent functional that can in principle be solved self-consistently via the optimized effective potential approach [21]. This is, however, numerically very demanding, and practical $\mathrm{EX}+\mathrm{cRPA}$ calculations are commonly performed in a postprocessing fashion, where single-particle orbitals

Published by American Physical Society under the terms of the Creative Commons Attribution 3.0 License. Further distribution of this work must maintain attribution to the author(s) and the published article's title, journal citation, and DOI.
PACS numbers: 31.15.ae, 31.15.vq, 31.15.xp, 71.15.Mb

from a self-consistent DFT calculation in the local-density approximation (LDA), generalized gradient approximations (GGAs), or alike, are used to evaluate both the EX and cRPA terms. Alternatively, one can formulate cRPA in terms of many-body perturbation theory (MBPT) based on a HF reference.

Throughout this Letter we will adopt the following nomenclature: $E^{F} @ \mathrm{SC}$ is the total energy of the functional $F$, evaluated with the orbitals of a self-consistent (SC) scheme, e.g., HF, or the Perdew-Burke-Ernzerhof (PBE) [22] GGA. The corresponding theoretical scheme is then labeled as $F @$ SC. We also use the letter "x" or "c" in front of $F$ or as a subscript of $E^{F}$ to refer to the exchange or correlation part of the scheme explicitly. The functional $F$ can be exactexchange, or additionally contain RPA correlation (EX+ cRPA), etc. For instance, $E^{\mathrm{EX}} @ \mathrm{HF}$ is the self-consistent Hartree-Fock energy, whereas the conventional RPA scheme based on PBE orbitals is referred to as (EX+cRPA)@PBE.

The original (EX+cRPA)@PBE and (EX+cRPA)@HF schemes both exhibit systematic underbinding for a large variety of systems, including covalent molecules [3], weakly bonded molecules [7,8], solids [11], and molecules adsorbed on surfaces [13-15]. Several attempts have been made to improve the accuracy of EX+cRPA. The earliest is the so-called RPA+ scheme [23], where a local correction at the LDA/GGA level is added to cRPA. More recent attempts add second-order screened exchange (SOSEX) $[9,24])$ to make the entire approach self-correlation free, or invoke cRPA in a range-separated framework where only the long-range part of cRPA is incorporated [7,8]. Among these, RPA+ improves total correlation energies considerably [25], but not binding energies [3]. The SOSEX correction performs well $[9,24]$ with considerable additional numerical effort. Range-separated RPA schemes also improve upon the standard EX+cRPA scheme $[7,8,16]$, however, at the price of introducing empirical parameters in the approach. 
In this Letter, we offer a new perspective, based on MBPT, for going beyond cRPA, and show that a simple modification of the standard EX+cRPA scheme leads to a significant accuracy increase for molecular binding energies. We first illustrate our key idea using the example of $\mathrm{Ar}_{2}$.

The (EX+cRPA)@PBE and (EX+cRPA)@HF binding energy curves for $\mathrm{Ar}_{2}$ are plotted in Fig. 1(a). Both schemes show a significant underbinding behavior compared to the reference curve modeled by Tang and Toennies [26] based on experimental data. To gain more insight into the origin of the underbinding, the EX+cRPA binding energies are decomposed into two contributions in Fig. 1(b): the exchange-only part and the remaining cRPA part. Inspection of the individual components reveals that $E_{\mathrm{c}}^{\mathrm{cRP}} @ \mathrm{HF}$ is (much) more repulsive than $E_{\mathrm{c}}^{\mathrm{cRPA}} @ \mathrm{PBE}$, whereas at the EX level $E^{\mathrm{EX}} @ \mathrm{PBE}$ is (much) more repulsive than $E^{\mathrm{EX}} @ \mathrm{HF}$. The fact that $E_{\mathrm{c}}^{\mathrm{cRPA}} @ \mathrm{PBE}$ is more attractive than $E_{\mathrm{c}}^{\mathrm{cRPA}} @ \mathrm{HF}$ is easy to rationalize by inspecting the corresponding frequency-dependent polarizabilities and the $C_{6}$ coefficients derived from them. Extensive benchmark calculations for 1225 molecular pairs [30] show that asymptotic $C_{6}$ dispersion coefficients derived from $E_{\mathrm{c}}^{\mathrm{cRPA}} @ \mathrm{HF}$ are systematically too small by approximately $40 \%$ [27], while this error is only $\sim 10 \%$ for $E_{\mathrm{c}}^{\mathrm{cRPA}} @ \mathrm{PBE}$. Adding $\Delta \mathrm{vdW}$ corrections in an attempt to reduce the remaining error in cRPA@PBE [31] only leads to minor changes in the binding energy at the equilibrium distance. What is more striking, however, is the considerable difference in binding energies at the EX level$E^{\mathrm{HF}} @ \mathrm{HF}-E^{\mathrm{EX}} @ \mathrm{PBE}$ [plotted also in Fig. 1(b) (red stars)]. It amounts to $\sim 6 \mathrm{meV}$ at the equilibrium distance and is thus close to the deviation of the (EX+cRPA)@PBE binding energy from the reference value.

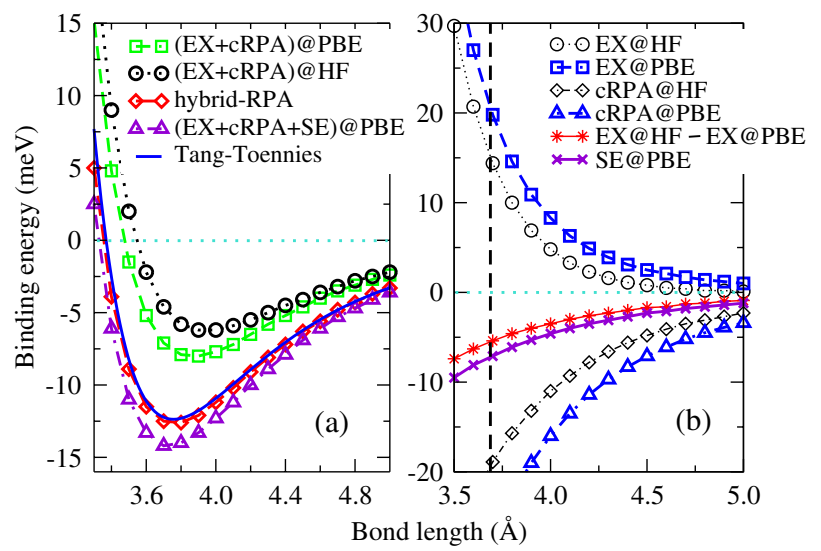

FIG. 1 (color online). (a) Binding energy curve for $\mathrm{Ar}_{2}$ computed with four RPA-based approaches, in comparison to the accurate reference curve by Tang and Toennies [26]. (b) Decomposition of the (EX+cRPA)@HF [(EX+cRPA)@PBE] binding energy of $\mathrm{Ar}_{2}$ into individual contributions: EX@HF (EX@PBE) and cRPA@HF (cRPA@PBE). The difference between EX@HF and EX@PBE, and the SE@PBE term, are also plotted. The vertical dashed line marks the equilibrium distance. Calculations are done using FHI-aims [27,28] and Dunning's aug-cc-pV6Z basis [29]. The basis set superposition error is corrected here and in the following.
From the viewpoint of Rayleigh-Schrödinger perturbation theory (RSPT), $E^{\mathrm{EX}} @ \mathrm{HF}$ and $E^{\mathrm{EX}} @ \mathrm{PBE}$ correspond to the sum of the zeroth and first-order terms in the perturbative expansions based on HF and PBE reference states, respectively [32]. The difference between $E^{\mathrm{EX}} @ \mathrm{HF}$ and $E^{\mathrm{EX}} @ \mathrm{PBE}$ must therefore be compensated by higherorder terms in the perturbation series since the final result should be independent of the reference state, if all terms were summed up. The next term in the series is the 2 ndorder correlation energy $E_{\mathrm{c}}^{(2)}$, to which only single and double excitation configurations contribute. Here we particularly examine the contribution of single excitations (SE) to $E_{\mathrm{c}}^{(2)}$, which can be expressed [32] as

$$
E_{\mathrm{c}}^{\mathrm{SE}}=\sum_{i}^{\mathrm{occ}} \sum_{a}^{\text {unocc }} \frac{\left|\left\langle\psi_{i}|\hat{f}| \psi_{a}\right\rangle\right|^{2}}{\epsilon_{i}-\epsilon_{a}} .
$$

Here $\psi_{i}$ and $\epsilon_{i}$ are the single-particle orbitals and orbital energies of the reference state, and $\hat{f}$ is the single-particle HF Hamiltonian - the Fock operator. A more detailed derivation of Eq. (1) is given in the supplemental material [33] (where we simply follow RSPT instead of the GörlingLevy PT [34]). As a consequence of the Brillouin theorem [32], $E_{\mathrm{c}}^{\mathrm{SE}}$ trivially vanishes for HF orbitals, but is in general nonzero for $\mathrm{KS}$ orbitals [33]. The contribution of $E_{\mathrm{c}}^{\mathrm{SE}}$ evaluated with PBE orbitals (referred to as SE@PBE) to the binding energy of $\mathrm{Ar}_{2}$ is also plotted in Fig. 1(b) (violet crosses). It amounts to $50 \%$ of the binding energy at the equilibrium distance, and is close in magnitude to the contribution from $E^{\mathrm{EX}} @ \mathrm{HF}-E^{\mathrm{EX}} @ \mathrm{PBE}$, and to the amount of underbinding in the original (EX+cRPA)@PBE scheme. We therefore propose a new scheme by adding $E_{\mathrm{c}}^{\mathrm{SE}}$ to $E^{\mathrm{EX}+\mathrm{cRPA}}$ (subsequently referred to as $\mathrm{EX}+\mathrm{cRPA}+\mathrm{SE}$ ). In Fig. 1(a) the resultant $(\mathrm{EX}+\mathrm{cRPA}+\mathrm{SE}) @ \mathrm{PBE}$ binding energy curve is also plotted, which improves considerably over the (EX+cRPA)@PBE results, and is in close agreement with the Tang-Toennies reference curve.

It appears that the quantitative agreement between $E_{\mathrm{c}}^{\mathrm{SE}}$ defined in Eq. (1) and $E^{\mathrm{EX}} @ \mathrm{HF}-E^{\mathrm{EX}} @ \mathrm{PBE}$ is a general feature. We found for a set of 50 atoms and molecules that the agreement typically ranges between $70 \%$ and $100 \%$, suggesting that replacing $E^{\mathrm{EX}} @ \mathrm{PBE}$ by $E^{\mathrm{EX}} @ \mathrm{HF}$ is an effective way to account for the SE contributions. This leads to a "hybrid-RPA" scheme, whose total energy is given by

$$
E^{\text {hybrid-RPA }}=E^{\mathrm{EX}} @ \mathrm{HF}+E_{\mathrm{c}}^{\mathrm{cRPA}} @ \mathrm{PBE},
$$

as an alternative to boost the accuracy of RPA. Figure 1(a) shows that the resultant binding energy curve is in almost perfect agreement with the reference curve.

At this point, it is illustrative to take a closer look at the individual contributions to $E^{\mathrm{EX}} @ \mathrm{HF}-E^{\mathrm{EX}} @ \mathrm{PBE}$. In Fig. 2 we further decompose the EX@HF and EX@PBE binding energies into their kinetic $\left(T_{s}\right)$, electrostatic $\left(E^{\text {elec }}\right.$, external potential energy and Hartree energy combined), and exchange components $\left(E_{\mathrm{x}}^{\mathrm{EX}}\right)$ for $\mathrm{Ar}_{2}$.

All three energy components behave quite differently for HF and PBE orbitals. The HF kinetic energy is purely 


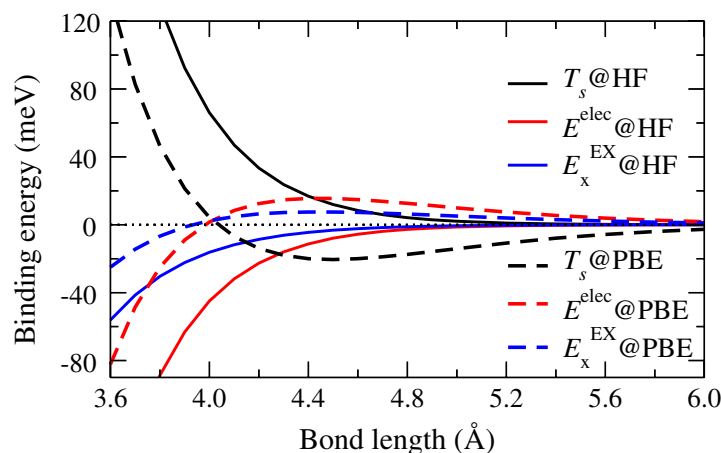

FIG. 2 (color online). Decomposition of the $E^{\mathrm{EX}} @ \mathrm{HF}$ and $E^{\mathrm{EX}} @ \mathrm{PBE}$ binding energies for $\mathrm{Ar}_{2}$ into their kinetic, electrostatic, and exchange components.

repulsive, whereas the $\mathrm{PBE}$ one exhibits spurious attraction at intermediate and large distances. The HF electrostatic and exact-exchange energies, on the other hand, are purely attractive and decay to zero from below, while the corresponding PBE ones become repulsive in the intermediate range and decay to zero from above at large distances. Since the PBE orbitals are much more delocalized than the HF ones, all three energy components decay much slower in $\mathrm{PBE}$ than in HF. The overall effect is that $E^{\mathrm{EX}} @ \mathrm{PBE}$ becomes significantly more repulsive than $E^{\mathrm{EX}} @ \mathrm{HF}$, resulting in the underbinding behavior of $(\mathrm{EX}+\mathrm{cRPA}) @ \mathrm{PBE}$. The more physical behavior of EX@HF at the EX level provides a sound basis for the systematic improvement from (EX+cRPA)@PBE to hybrid-RPA.

Indeed, the exceptional performance of the hybrid-RPA and $(\mathrm{EX}+\mathrm{cRPA}+\mathrm{SE}) @ \mathrm{PBE}$ schemes for rare-gas dimers carries over to many other molecular systems. As a second example we show results for the $\mathrm{N}_{2}$ molecule adsorbed on benzene $\left(\mathrm{N}_{2} @\right.$ benzene), which is an important model system for studying molecular adsorption on graphene and graphite surfaces [35]. We consider two possible configurations: $\mathrm{N}_{2}$ placed parallel or perpendicular to the benzene plane. A successful theoretical approach for this system must be able to describe the delicate balance between electrostatic and dispersion interactions. We use FHI-aims (Fritz Haber Institute $a b$ initio molecular simulations) $[27,28]$ and numeric atom-centered orbital basis $(6 s 5 p 4 d 3 f 2 g$ for $\mathrm{C}, \mathrm{O}, \mathrm{N}$, and $5 s 3 p 2 d 1 f$ for $\mathrm{H})$ augmented with Gaussian diffuse functions from aug-cc-pV5Z to achieve convergence of the binding energy to within $1 \mathrm{meV}$. The results shown in Fig. 3 are very similar to the case of rare-gas dimers: (EX+cRPA)@ $\mathrm{HF}$ and $(\mathrm{EX}+\mathrm{cRPA}) @ \mathrm{PBE}$ underbind significantly at the equilibrium distance, while hybrid-RPA and (EX+cRPA+SE)@PBE bring the binding energy into much closer agreement with the reference curve computed with the coupled cluster method including single, double, and perturbative triple excitations [CCSD(T)] [35]. In contrast, the traditional MP2 method vastly overbinds the system.

Finally we examine the performance of hybrid-RPA and $(\mathrm{EX}+\mathrm{cRPA}+\mathrm{SE}) @ \mathrm{PBE}$ for the S22 database of Jurečka

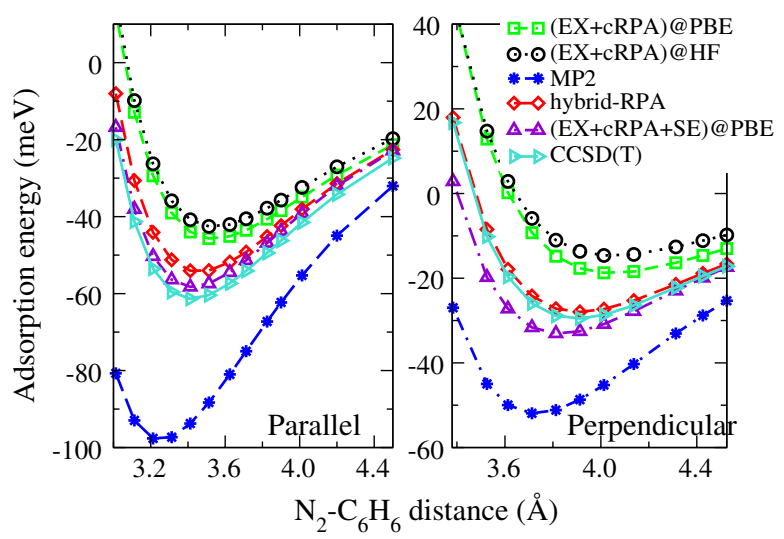

FIG. 3 (color online). Binding energies of the parallel and perpendicular configuration of $\mathrm{N}_{2}$ @benzene as a function of the $\mathrm{N}_{2}-\mathrm{C}_{6} \mathrm{H}_{6}$ center-of-mass distance, calculated by different RPA-based approaches as well as MP2, in comparison to reference $\operatorname{CCSD}(\mathrm{T})$ results from Ref. [35].

et al. [36], which represents a balanced benchmark set for noncovalent interactions. The molecular dimers in this database can be divided into three groups of different bonding types: hydrogen bonded, dispersion bonded, and mixed complexes. We note that RPA in a range-separated framework has been applied to the S22 database very recently [16]. In Fig. 4 we plot the deviation from the $\operatorname{CCSD}(\mathrm{T})$ reference values [37] for the binding energies of the S22 molecules [36] for four RPA-based approaches and MP2. The basis set type and quality are the same as for $\mathrm{N}_{2} @$ benzene. A detailed error analysis is presented in Table I.

We observe that the standard (EX+cRPA)@PBE scheme systematically underbinds all complexes. $(\mathrm{EX}+\mathrm{cRPA}) @$ HF performs even worse for dispersion and mixed bonding, but better for hydrogen bonding. The latter case can be explained by the fact that the better performance of EX@HF dominates over the bad performance of cRPA @HF for hydrogen-bonded systems. Again hybrid-RPA and $(\mathrm{EX}+\mathrm{cRPA}+\mathrm{SE}) @ \mathrm{PBE}$ correct the underbinding

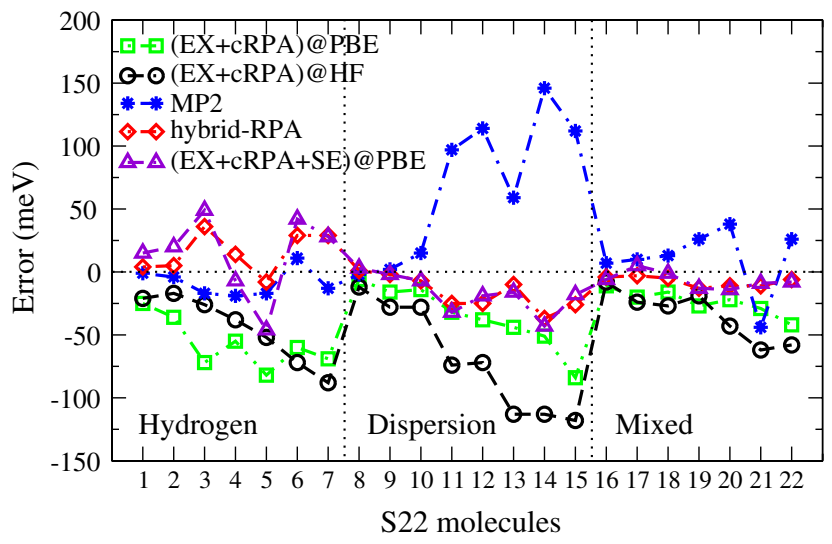

FIG. 4 (color online). Deviation from the $\operatorname{CCSD}(\mathrm{T})$ reference values [37] for the binding energies of the S22 database [36] for RPA-based approaches as well as MP2. Positive errors correspond to overbinding and negative ones to underbinding. 
TABLE I. Mean absolute error (in meV) and mean absolute percentage error (in parenthesis) of different RPA-based approaches for the S22 database [36]. CCSD(T) extrapolated to the complete basis set limit [37] is taken as reference.

\begin{tabular}{lccc}
\hline \hline & H bond & Dispersion & Mixed \\
\hline (EX+cRPA)@HF & $45(8.5 \%)$ & $70(43.9 \%)$ & $34(20.9 \%)$ \\
(EX+cRPA)@PBE & $57(11.2 \%)$ & $36(21.8 \%)$ & $24(15.0 \%)$ \\
(EX+cRPA+SE)@PBE & $30(6.0 \%)$ & $18(12.0 \%)$ & $8(5.5 \%)$ \\
Hybrid-RPA & $18(3.0 \%)$ & $17(10.0 \%)$ & $8(5.1 \%)$ \\
\hline \hline
\end{tabular}

behavior of the standard EX+cRPA scheme, and improve the accuracy considerably. The hybrid-RPA scheme yields a mean absolute error (MAE) of $14 \mathrm{meV}$. The performance of $(\mathrm{EX}+\mathrm{cRPA}+\mathrm{SE}) @ \mathrm{PBE}$ is very similar to hybrid-RPA for dispersion and mixed bonding, albeit somewhat worse for hydrogen bonding. However, the mean absolute percentage error for hydrogen bonding (6\%) is still quite small. The accuracies achieved here compare favorably to the recently developed vdW functional (vdW-DF) [38], where the MAE for the PBE-based vdW-DF results for S22 [39] is $54 \mathrm{meV}$. We also note that for covalent molecules the accuracies in the atomization energies are improved considerably by the two schemes. For instance, the MAE of the atomization energies of the G2-I set is reduced from 10.5 to $6.2 \mathrm{kcal} / \mathrm{mol}$ by $(\mathrm{EX}+\mathrm{cRPA}+\mathrm{SE}) @ \mathrm{PBE}$ and $6.3 \mathrm{kcal} / \mathrm{mol}$ by hybrid-RPA.

To summarize, we have unraveled the origin of the underbinding that plagues the standard (EX+cRPA)@PBE scheme, which is mostly due to the too-repulsive nature of $E^{\mathrm{EX}} @ \mathrm{PBE}$ rather than the (slight) underestimation of the long-range dispersion force by $E_{\mathrm{c}}^{\mathrm{cRPA}} @$ PBE. This problem can be largely solved either by replacing $E^{\mathrm{EX}} @ \mathrm{PBE}$ by the self-consistent $\mathrm{HF}$ energy $E^{\mathrm{EX}} @ \mathrm{HF}$ or by adding a SE correction to the standard (EX+cRPA)@PBE approach. In particular, $(\mathrm{EX}+\mathrm{cRPA}+\mathrm{SE}) @ \mathrm{PBE}$ is a well-defined parameter-free scheme in which the SE term does not add any significant computational cost to the approach. In addition, the SE correction is compatible with other beyond-RPA schemes like RPA+ or SOSEX. We also like to emphasize that in both schemes cRPA is evaluated with KS orbitals, which is essential for producing quantitatively correct asymptotics for vdW bonded systems. Despite its success for describing vdW and covalently bonded molecules, one obvious deficiency of the 2nd-order SE as given by Eq. (1), however, is that it is not well behaved for systems with vanishing gaps. In such cases, we propose to "renormalize" the SE contribution via a resummation of a geometrical series of higher-order diagrams involving single excitations (in the spirit of cRPA). This leads to additional terms in the denominator of Eq. (1) which prevent the possible divergence even when the KS gap closes. A brief derivation of this renormalized SE (RSE) scheme is presented in the supplemental material [33]. And we note in passing that SE and RSE are invariant to unitary transformations of the occupied or virtual orbitals [27,33]. Further details and benchmark calculations will be published elsewhere [27].

[1] D. Bohm and D. Pines, Phys. Rev. 92, 609 (1953).

[2] M. Gell-Mann and K. A. Brueckner, Phys. Rev. 106, 364 (1957).

[3] F. Furche, Phys. Rev. B 64, 195120 (2001).

[4] M. Fuchs and X. Gonze, Phys. Rev. B 65, 235109 (2002).

[5] F. Furche, J. Chem. Phys. 129, 114105 (2008).

[6] G. E. Scuseria, T. M. Henderson, and D. C. Sorensen, J. Chem. Phys. 129, 231101 (2008).

[7] B. G. Janesko, T. M. Henderson, and G. E. Scuseria, J. Chem. Phys. 130, 081105 (2009).

[8] J. Toulouse et al., Phys. Rev. Lett. 102, 096404 (2009).

[9] J. Paier et al., J. Chem. Phys. 132, 094103 (2010).

[10] A. Marini, P. García-González, and A. Rubio, Phys. Rev. Lett. 96, 136404 (2006).

[11] J. Harl and G. Kresse, Phys. Rev. Lett. 103, 056401 (2009).

[12] D. Lu, Y. Li, D. Rocca, and G. Galli, Phys. Rev. Lett. 102, 206411 (2009).

[13] M. Rohlfing and T. Bredow, Phys. Rev. Lett. 101, 266106 (2008).

[14] X. Ren, P. Rinke, and M. Scheffler, Phys. Rev. B 80, 045402 (2009).

[15] L. Schimka et al., Nature Mater. 9, 741 (2010).

[16] W. Zhu et al., J. Chem. Phys. 132, 244108 (2010).

[17] J.F. Dobson, in Topics in Condensed Matter Physics, edited by M. P. Das (Nova, New York, 1994).

[18] C. Møller and M. S. Plesset, Phys. Rev. 46, 618 (1934).

[19] D. C. Langreth and J. P. Perdew, Phys. Rev. B 15, 2884 (1977).

[20] W. Kohn and L. J. Sham, Phys. Rev. 140, A1133 (1965).

[21] S. Kümmel and L. Kronik, Rev. Mod. Phys. 80, 3 (2008).

[22] J. P. Perdew, K. Burke, and M. Ernzerhof, Phys. Rev. Lett. 77, 3865 (1996).

[23] Z. Yan, J. P. Perdew, and S. Kurth, Phys. Rev. B 61, 16430 (2000).

[24] A. Grüneis et al., J. Chem. Phys. 131, 154115 (2009).

[25] H. Jiang and E. Engel, J. Chem. Phys. 127, 184108 (2007).

[26] K. T. Tang and J. P. Toennies, J. Chem. Phys. 118, 4976 (2003).

[27] X. Ren et al. (to be published).

[28] V. Blum et al., Comput. Phys. Commun. 180, 2175 (2009).

[29] J. T. H. Dunning, J. Chem. Phys. 90, 1007 (1989).

[30] A. Tkatchenko and M. Scheffler, Phys. Rev. Lett. 102, 073005 (2009).

[31] A. Tkatchenko et al., J. Chem. Phys. 131, 094106 (2009).

[32] A. Szabo and N. S. Ostlund, Modern Quantum Chemistry: Introduction to Advanced Electronic Structure Theory (McGraw-Hill, New York, 1989).

[33] See supplemental material at http://link.aps.org/ supplemental/10.1103/PhysRevLett.106.153003 for the derivations of SE and renormalized SE schemes.

[34] A. Görling and M. Levy, Phys. Rev. B 47, 13105 (1993).

[35] A. Tkatchenko et al. (to be published).

[36] P. Jurečka et al., Phys. Chem. Chem. Phys. 8, 1985 (2006).

[37] T. Takatani et al., J. Chem. Phys. 132, 144104 (2010).

[38] M. Dion et al., Phys. Rev. Lett. 92, 246401 (2004).

[39] A. Gulans, M. J. Puska, and R. M. Nieminen, Phys. Rev. B 79, 201105 (2009). 